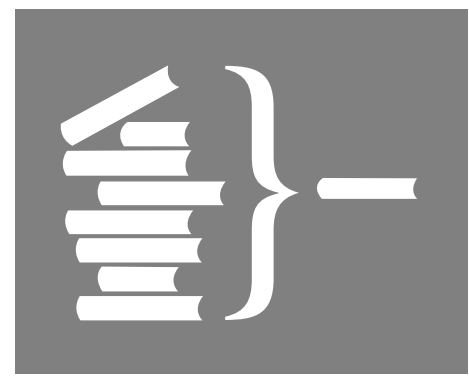

\title{
The Development of Live Attenuated Cold-adapted Influenza Virus Vaccine for Humans
}

\author{
Hunein F. Maassab ${ }^{1 *}$ and Martin L. Bryant ${ }^{2}$ \\ ${ }^{1}$ Department of Epidemiology, School of Public Health, University of Michigan, \\ 109 Observatory Street, Ann Arbor, Michigan 48109, USA \\ ${ }^{2}$ Aviron, 297 North Bernardo Ave, Mountain View, CA 94043, USA
}

\begin{abstract}
SUMMARY
A procedure to attenuate live influenza virus of type $A$ and type $B$ was developed using adaptation of the virus to grow at $25^{\circ} \mathrm{C}$ (cold adaptation; ca). Through a series of stepwise passages, two stable mutants were obtained and designated as 'Master' strains, one for type A influenza virus (A/Ann Arbor/6/60-H2N2) and one for type B influenza virus (B/Ann Arbor/1/66). These mutants were used in genetic reassortment using either the classical method or more recently described reverse genetics to update the relevant surface antigens of the circulating strains of influenza virus. The derivation is based on the concept of $6 / 2$ where 6 signifies the six internal genes of the master strain and 2 refers to the two genes coding for the two surface glycoproteins HA and NA of the circulating influenza virus. The advantages of this vaccine were demonstrated to be (1) proper level of attentuation, (2) non-transmissibility, (3) genetic stability, (4) presence of the ca and ts markers and (5) immunogenicity involving both local and the cell-mediated immune responses. The clinical trials in infants, children, adults and elderly have provided the necessary data for eventual licensing of this vaccine. The ease of administration (intranasal) safety and high efficacy make this vaccine suitable to prevent influenza virus infection in all age groups. Copyright (C) 1999 John Wiley \& Sons, Ltd.
\end{abstract}

Accepted 20 January 1999

\section{INTRODUCTION}

Prophylaxis of influenza virus has been a recurring public health concern for many years with frequent reexamination of variable vaccines and policies for their use. Outbreaks and epidemics of influenza virus type A and B exhibit definite excess mortality (especially among the elderly) and considerable morbidity (especially among school-age children) which are regularly observed during the influenza season. In the case of a disease such as influenza, it has become recognised that antigenic changes in the causative viruses impose a profound handicap to the development of a sound system of immunisation. The time factor alone $(\sim 14$ days $)$ has

${ }^{*}$ Correspondence to: Dr H. F. Maassab, Department of Epidemiology, School of Public Health, University of Michigan, 109 Observatory Street, Ann Arbor, Michigan 48109, USA.

Abbreviations used: ca, cold-adapted; CAIV-T, cold-adapted influenza vaccine-trivalent; $C R$, cold-reassortant; FDA, Federal Drug Administration; Ha, haemagglutinin; IgA, immune globulin A; PCKC, Primary chick kidney cells; $\mathrm{Na}$, neuraminidase; $\mathrm{NIH}$, National Institutes of Health; PFU, plaque forming unit; SPAFAS, pathogenfree; ts, temperature sensitive; vac, vaccine. suggested that inactivated vaccine will never suffice to provide the limitation in the transmission of virus which could furnish the power to prevent epidemic spread. For these reasons, research and clinical testing is continuing with the aim of identifying an effective and safe live influenza virus vaccine for use in humans. ${ }^{1-6}$

\section{HISTORICAL BACKGROUND}

The approach to prevention of influenza by vaccination is based on several observations. First, recovery from infection is accompanied by antibody development and resistance to reinfection. Second, in experimental animals antibody production and immunity can be induced by para respiratory injection of active or inactive virus. Third, circulating antibody levels similar to those observed in convalescence can be obtained in humans by vaccination, presumably reflecting an accompanying immunity. Vaccination enhances the neutralising capacity of nasal secretions in man, thus providing increased protection to the susceptible cells of the respiratory 
Table 1. Characteristics of persons considered high risk

1. Chronic debilitating disease, e.g. chronic cardiovascular, pulmonary, renal, or metabolic disorders.

2. Rheumatic heart disease, especially those with mitral stenosis.

3. Other cardiovascular disorders, especially those with evidence of frank or incipient cardiac insufficiency.

4. Chronic bronchopulmonary disease, diabetes mellitus or Addison's disease.

5. Pregnant women.

6. Persons in older age groups, those over 45 and, particularly, those over 65 years of age who contribute most to excess mortality.

mucosa. Vaccination has been shown to prevent the severe viral pneumonia produced in ferrets by welladapted strains of virus even though intranasal challenge may induce fever and damage to the ciliated epithelium, ${ }^{7,8}$

A number of early studies with active or inactive virus from various sources demonstrated that vaccines were immunogenic in humans, but the results of field prophylaxis were inconsistent, owing partly to low incidence of disease, inconstant potency of vaccine and strain variation. The incidence of disease in the vaccinated was inversely related to the level of circulating antibody to the epidemic strain; this observation has been well substantiated. ${ }^{9}$ The effect of vaccine became evident 7 to 10 days after administration. In some of the study units streptococcal infections were prevalent at the time influenza appeared, without creating significant complications. Other observations indicated that influenza was significantly reduced in institutional populations vaccinated a year earlier. The ability of vaccines to reduce severity of illness has been shown in experimental animals and in induced infections of human. On this basis it might be expected that even though moderate fever and local respiratory signs might occur, circulating antibodies would reduce the likelihood of lower respiratory involvement. $^{10-13}$

Based on the knowledge of strain variation and the variation in antibody patterns of the different age groups in the population, a continued objective has been to create a vaccine containing a pool of antigens including those known to have been prominent in epidemic strains of influenza virus. Moreover, secondary antigens contained within such a compound vaccine could potentially stimulate a broad antibody response against a wide range of other strains, just as human sera may, after repeated infections, contain antibody to strains which have not yet been encountered epidemically by the younger age groups. The 1957 experience emphasised that the dominant antigen of an earlier strain may return to epidemic circulation when the population loses its immunity by aging, death and a growing replacement by susceptibles. ${ }^{8,14,15}$ A compound vaccine could also provide conditioning to varied antigens early in life which could be boosted promptly by subsequent vaccination to estab- lish an enhanced broad resistance rather than acquiring it by repeated infections. Obviously, this approach requires a comprehensive immunologic effort and flexibility in formulation of vaccines, but present knowledge suggests it has a reasonable potential for addressing the great antigenic variability in influenza viruses.16-18

Limitations to this approach include the fact that vaccine does not prevent respiratory disease caused by other pathogens and by exaggerated talk of reactions. The duration of protection is not fully established, and annual vaccination is an inconvenience. Production of vaccine has not been sufficiently great to permit uniform vaccination annually. With consideration of the above factors, the Surgeon General's United States Public Health Service Advisory Committee on Influenza recommended annual vaccination of certain groups of persons shown by experience to be at high risk of severe or fatal illness with influenza. ${ }^{19-22}$

Epidemics may be so extensive as to disrupt normal community operation and strain its facilities. Consequently, personnel of community services-medical and health, utilities, safety, education and communicationsshould be protected. Vaccination of groups of industrial workers has been shown to be of benefit. ${ }^{5}$ The families of the people listed in Table 1, especially young children, could also benefit from vaccination, emphasising that influenza vaccine ought ordinarily to be considered for group administration. ${ }^{23,24}$ Vaccinations of school populations would serve to reduce the large impact which that segment of the population contributes to the community load of illness and its dissemination. The family can be considered the basic group for action. Thus, with the probability of epidemic involvement, no group is exempt from consideration at the community level. Vaccine has been used uniformly in the military of the United States for an extended period. The advantages are seen in the low incidence of disease in service units and installations when high levels are occurring in civilian populations. For example, in 1960, little or no epidemic influenza was detected in posts under close surveillance, while the civilian epidemic was pronounced. ${ }^{25}$

Another area of advance has been the demonstration that preparations of purified viral haemagglutinin are as effective as whole virus in the stimulation of antibody in 
Table 2. Criteria for Candidate vaccine

1. A reliable and reproducible method of attenuation (e. g. cold-adaptation at $25^{\circ} \mathrm{C}$.

2. Availability of an animal model to evaluate pathogenicity prior to use in man (ferret model).

3. Availability of a marker system, the cold-adapted (ca) and the temperature-sensitive markers (ts) to trace the vaccine in laboratory and field studies.

4. Non-transmissibility is an important factor from the point of view of minimising reversion and the uncontrolled spread in the community.

5. Administration to man by the natural route of transmission (respiratory tract, nose drops).

6. Genetic stability, since live vaccine which has the potential to revert to virulence would not be acceptable and would not be licensed for use in man.

children and adults. Indications are that the released viral antigens may induce broader immune responses than when they are contained in the intact virus particle. Importantly, it was observed that children tolerated, without local or febrile reaction, much larger doses of the viral subunits than of whole virus. These materials offer many potential advantages to wider application of vaccine.

\section{LIVE VIRUS VACCINE}

It has long been recognised that live attenuated influenza virus vaccine offers a potential advantage over inactivated vaccine. Such a vaccine has been developed through the process of adaptation at suboptimal temperatures $\left(25^{\circ} \mathrm{C}\right)$, a technique described as cold-adaptation.

The adaptation of influenza virus of type $A$ and type $B$ to growth $25^{\circ} \mathrm{C}$ has been found to be a reliable technique for the development of attenuated viruses as candidates for live virus vaccine for use in man. ${ }^{26-30}$ The extensive experience acquired in our laboratory in the past 20 years in the development of cold-adapted (ca) influenza virus vaccine allows us to conclude that cold-adaptation using recent human isolates can be considered a reliable technique to attenuate influenza virus. ${ }^{31}$

\section{Derivation of 'Master' strains for type A and type $B$ influenza virus}

Domingo $e t$ al. ${ }^{32}$ emphasised that RNA viruses in general exhibit a high degree of mutation and variability. For example, influenza viruses exhibit antigenic variation through either genetic reassortment with animal virus or antigenic variation influenced by the host immune response or by antiviral drugs. Thus, quasispecies with changes in antigenic specificity may emerge which are resistant to the selective pressure of the host necessitating periodic update of vaccines or changes in drug regimen. Studies in our laboratories provided evidence that temperature of incubation of infected cells can be a factor in the alteration of virulence of mammalian viruses grown in cell culture. Increased virulence of certain viruses was correlated with their ability to grow at a temperature above the physiological range $\left(38^{\circ}-39^{\circ} \mathrm{C}\right)$. In contrast, adaptation of the virus to growth at suboptimal temperature $\left(25^{\circ} \mathrm{C}\right)$ resulted in decreased virulence or attenuation. Thus, virulence can be quantified and is a quality that can be added or lost experimentally from a virus strain. ${ }^{33,34}$ To develop an attenuated live influenza virus vaccine we adapted influenza virus of type $A$ or $B$ to grow to a maximum titre at $25^{\circ} \mathrm{C}$ in cultures of primary chick kidney cells and to derive ca mutants. ${ }^{34}$ The criteria for a candidate vaccine are listed in Table 2 and could be applied to any other viral pathogen of human importance.

The process of cold-adaptation involved two different processes. The first used stepwise adaptation of influenza virus until a maximum titre at $25^{\circ} \mathrm{C}$ was achieved. The second procedure used direct passage of the virus from the normal physiological temperature of $35-36^{\circ} \mathrm{C}$ to the suboptimal temperature of $25^{\circ} \mathrm{C}$ (cold-adaptation) followed by selection of the cold variant. The in vivo and in vitro characterisation of the derived cold-adapted variants demonstrated that they were attenuated for humans, were immunogenic and possess two markers, the ca and ts phenotypes, which correlated with the loss of virulence. The type A cold variant A/Ann Arbor/6/60-H2N2 and the type $B$ influenza virus $B / A A / 1 / 66$ upon administration intranasally to ferrets and to man were shed at low levels in pharyngeal secretions, with no reversion or transmission of the shed virus. Molecular characterisation of these viruses based on the migration of the RNA segments, using polyacrylamide gel electrophoresis, showed that $\mathrm{A} / \mathrm{AA} / 6 / 60-\mathrm{H} 2 \mathrm{~N} 2$ and $\mathrm{B} / \mathrm{AA} / 1 / 66$ exhibited changes in the migration of their segments, when compared with the original unadapted segments, implying that mutation(s) change in lesion(s) occurred upon coldadaptation, which can account for their genetic stability. It is for this reason that the cold-adapted lines $\mathrm{A} / \mathrm{AA} / 6 /$ $60-\mathrm{H} 2 \mathrm{~N} 2 \mathrm{~B} / \mathrm{Ann}$ Arbor/1/66 were designated as the 'Master' strains or donor of all attenuating genes for use in the derivation of cold-reassortant vaccine lines to be used in man. However, the stepwise cold-adaptation procedure was not practical for updating the live vaccines in the event of shift or drift in the circulating viruses (6-8 months). ${ }^{33}$ 
Table 3. A flow diagram for the derivation of type A cold reassortant vaccine

Step 1. Wild type parent with relevant surface antigens was plaque-purified twice at $39^{\circ} \mathrm{C}$ to enhance its virulence and to achieve a genetic homogeneity of the virulent wild-type before the reassortant procedure.

Step 2. The 'Master' strain cold-adapted A/AA/6/60-H2N2 7PI with three passages in SPAFAS embryonated eggs is the attenuated parent.

Step 3. Co-infection in primary chick kidney cells (PCKC) with multiplicity of infection of 5 plaqueforming units per $\mathrm{mL}$ for each parent $-48 \mathrm{~h}$ incubation $\rightarrow$ maximum cytolysis. The cultures are frozen, thawed, centrifuged, and the supernatant was passaged.

Step 4. Passaged $2 \mathrm{X}$ at $33^{\circ} \mathrm{C}$ in PCKC in presence of ferret immune serum to cold-adapted strain.

Step 5. Passaged $\mathrm{IX}$ at $33^{\circ} \mathrm{C}$ in PCKC in absence of ferret immune serum.

Step 6. $\quad$ Passaged IX in SPAFAS embryonated hens' eggs at $33^{\circ} \mathrm{C}$.

Step 7. Plaque titration at $33^{\circ} \mathrm{C}$ in PCKC - selection of 20 clones for characterisation.

Step 8. Selection of a clone as a seed vaccine on the basis that six internal genes were derived from the 'Master' strain.

Step 9. Plaque to plaque purification $(3 X)$ at $33^{\circ} \mathrm{C}$ in PCKC.

Step 10. Passage in SPAFAS embryonated hens' eggs for production of seed virus for distribution and for production of volunteer pool.

Step 11. Choice of a cold-reassortant clone is based on

(i) having six genes from the 'Master' strain;

(ii) ts and ca markers;

(iii) no reactogenicity in ferrets.

Step 12. The procedure was modified for the derivation of type B cold-adapted reassortant vaccine using the 'Master' strain B/Ann Arbor/1/66-7PI-SE3 by lowering the temperature at $25^{\circ} \mathrm{C}$ only during the whole process of cloning. This was necessitated by the similarities of surface antigens of influenza $\mathrm{B}$. Thus, the selection pressure provided by using a $25^{\circ} \mathrm{C}$ incubation temperature yielded not only a higher frequency in the derivation of $6 / 2$ reassortant vaccines but also shortened the time required by allowing us to screen 100 clones for the appropriate vaccine line.

\section{The $6 / 2$ formula for the derivation of live cold-reassortant $(\mathrm{CR})$ vaccine lines}

Cold reassortants possessing the six internal genes from the cold-adapted "Master" donor parent and the two genes which code for the two surface glycoproteins of the circulating wild type parent will be referred to in this report as the $6 / 2$ cold reassortant vaccine. ${ }^{31,33,34}$ Evaluation of the $6 / 2$ reassortant vaccines in an animal model (ferrets) showed attenuation, immunogenicity and acceptability similar to their donor attenuated viruses (A/Ann Arbor/6/60 and B/Ann Arbor/1/66). Thus, it can be stated that laboratory criteria are available to identify, predict and monitor the development and characterisation of live influenza virus prior to administration to man (Table 3). These laboratory criteria are offered for reproducibility and rapidity of derivation of live influenza virus prior to administration to man including the response to new epidemic strains. In addition, gene analysis of these vaccine candidates has allowed us to monitor the $C R$ during all phases of vaccine development from the laboratory to the manufacturer to use in man as well as for monitoring isolates from the field trials. The reproducibility of these attenuated $6 / 2$ reassortants can be consistently achieved. For the past 12 years, these live influenza virus cold reassortant vaccines, with $\mathrm{HA}$ and NA genes from different wild type viruses, were proven to be predictably attenuated for man, immunogenic, did not spread to contacts, had varying degrees of potency in seronegative and seropositive vaccinees and had genetic stability demonstrated through retention of the ca and ts phenotypes in all isolates obtained from trial in volunteers. In recent studies in volunteers, the efficacy of a live attenuated cold reassortant influenza vaccine with a $6 / 2$ gene composition was compared with a licensed inactivated vaccine. The results were encouraging and showed advantages (Table 4 ) of the live vaccine with regard to lack of reactogenicity, natural route of administration and protection upon wild type challenge. ${ }^{24,35,36}$

Studies of these influenza virus live cold reassortant vaccines in seronegative children indicate that the transfer of the six genes from the 'Master strains' A/Ann Arbor/6/60-H2N2 or B/Ann Arbor/1/66 cold-adapted parents to different wild type parents with relevant surface antigens have reproducibly conferred significant benefits. These include efficient infection, an acceptable level of attenuation, significant protective response to artificial or natural challenge, lack of transmissibility and no evidence of reversion with retention of the ca and ts phenotypes in the shed virus, implying genetic stability. Nasal antibody and cell-mediated immunity was also demonstrated. One of the potential advantages of an attenuated live influenza virus vaccine derives from limited replication in the infected host producing adequate stimulation of both humoral and local secretory immunoglobins $(\operatorname{Ig} \mathrm{A})$ as well as cell-mediated immune responses at the local site of viral administration. ${ }^{37}$ 
Table 4. Advantages of the cold-adaptation methods in the development of live influenza virus vaccines for man

1. Availability of laboratory guidelines for assessment of virulence.

2. Availability of cold-adapted 'Master Strain' A/AA/6/60-H2N2 and B/AA/1/66 as donors of attenuated genes.

3. Rapidity in the development of cold reassortant clones with the desired gene constellation.

4. Presence of ts and ca markers.

5. Genetic stability.

6. Reproducibility in attenuation and immunogenicity for man.

7. Lack of transmission.

Additional advantage was demonstrated when Wright and co-workers showed for amnestic recall (induction of memory) of influenza-specific IgA antibody in those children with a wild type natural infection or in children administered the attenuated live influenza A virus compared with children with no prior experience of influenza. $^{38}$ Thus, both cell-mediated immunity (local) and humoral antibodies appear to be mediators of immune protection against influenza infection and disease. The examples cited above emphasise that these live attenuated $\mathrm{CR}$ vaccines offer an advantage by inducing both types of antibodies, by minimising shedding and finally by their inability to spread.

\section{CURRENT STATUS}

The current clinical trial programme being conducted by Aviron in collaboration with the NIH is designed to support application to the FDA for use of a new influenza vaccine in children, adults, and the elderly. ${ }^{39}$ The product is a trivalent formulation (two type $A$ strains and one type B strain) of the live, attenuated, cold-adapted influenza vaccine (CAIV-T) delivered intranasally as a spray. This vaccine provides an alternative to the injectable vaccine for healthy and high risk children and adults, and could be co-administered with the injectable vaccine in the elderly.

The live, attenuated, ca influenza vaccine technology of H.F. Maassab was licensed to Aviron by the University of Michigan and NIH in 1995 after more than 90 clinical trials of ca vaccine strains had been were conducted by the Vaccine Treatment and Evaluation Units (VTEU) of the National Institute of Allergy and Infectious Diseases (NIAID) of the NIH. More than 15000 volunteers aged from 2 months to over 100 years received monovalent or bivalent formulations of the vaccine while trivalent formulations were tested in approximately 350 adults and 200 children. The results of these clinical trials consistently showed that the ca vaccine was safe and well tolerated, immunogenic, genetically stable, and non-transmissible. Efficacy of the monovalent and bivalent formulations of the ca vaccine were demonstrated in multiple experimental challenge studies and in clinical field trials, including the Vanderbilt study which was conducted over four influenza seasons from 1986 to 1990 in 5000 children and adults. ${ }^{40}$

Aviron and the NIH initially conducted a double blind, placebo controlled study of CAIV-T to demonstrate efficacy of the intranasal trivalent formulation in healthy adults. ${ }^{41}$ Volunteers were randomised to receive either CAIV-T, the inactivated injectable vaccine, or placebo and then challenged one month later with the wildtype influenza virus to which they had originally been shown to be seronegative. The reduction in laboratorydocumented influenza illness due to the wildtype H1N1, $\mathrm{H} 3 \mathrm{~N} 2$ or B strains compared with placebo was statistically significant for CAIV-T, $85 \%(P=0.001)$, and the injectable vaccine, $71 \%(P=0.01)$.

Following routine testing of the intranasal CAIV-T vaccine for safety, immunogenicity, and determination of dose in children, ${ }^{41}$ Aviron and the NIH conducted a multicentre, randomised, double-blind, placebo-controlled field efficacy trial in 1602 children, 17-71 months old during the 1996-97 influenza season. The efficacy against $\mathrm{A} / \mathrm{H} 3 \mathrm{~N} 2$ was $95 \%$ and the efficacy against influenza B was 91\%. In addition, there was a $30 \%$ reduction in febrile otitis media in the children vaccinated with CAIV-T. ${ }^{42,43}$.

Of the 1602 children enrolled in year one of the study, 1358 returned in year two (1997-98 influenza season). They remained in their randomised groups and were vaccinated with a single dose of CAIV-T or a placebo spray. Results from the second year of the double-blind, placebo-controlled efficacy study showed that CAIV-T provided $87 \%$ protection against all culture-confirmed influenza including the A/Sydney strain which was not in the vaccine. The A/Sydney influenza strain was not included in the 1997-98 formulation because CAIV-T was designed to match the influenza vaccine marketed that year. In the 1358 participants, there were 5 cases of influenza due to influenza strains included in the vaccine and 66 cases caused by the A/Sydney strain. Only $2 \%$ of children vaccinated with CAIV-T (15 out of 917) experienced culture-confirmed influenza, all of which was attributable to the A/Sydney strain, while $13 \%$ of the placebo recipients (56 out of 441) experienced cultureconfirmed influenza. The difference between these two 
influenza attack rates was used to calculate the overall protection rate of $87 \%$. The incidence of pneumonia and other lower respiratory diseases was also reduced in the vaccinees, compared with placebo recipients. Eight children in the placebo group developed influenza-related wheezing, bronchitis or pneumonia. All of these were due to the A/Sydney strain. No children who received CAIV-T experienced lower respiratory complications. The year two data also showed that CAIV-T provided $94 \%$ protection against influenza-related otitis media $(2$ cases in the vaccine group vs. 17 cases in the placebo group). ${ }^{43}$.

Because the ca influenza vaccine is delivered as a nasal spray, it should provide the first practical way to immunise children on an annual basis. Children are an important target because, while the elderly experience the greatest mortality from the annual influenza epidemic, much of the morbidity and illness occurs in young children. Children are also thought to be important to the spread of influenza in the population. In addition to its proposed use in physician(s) offices, the nasal delivery of this vaccine should enable it to be administered by adults without special medical training, so that it will be practical to consider delivery in pharmacies, schools, day care centres, and possibly in the home.

Clinical trials to obtain effectiveness data in healthy working adults, and safety data in asthmatic children are in progress. Additional trials are planned for the 1998-99 influenza season to study herd immunity in children and efficacy of co-administration with injected influenza vaccine in high risk adults.

Of interest, a novel method that uses the reverse genetics ${ }^{4445}$ technology to generate the vaccine strains each year (6:2 reassortants) has recently been developed $^{45}$. It is now possible to selectively introduce the HA and NA gene segments of wild type influenza A or B virus directly into the cold-adapted $A$ and $B$ master strains without in vitro propagation of wild type influenza virus. This method should significantly shorten the time required to prepare 6:2 reassortants for the annual vaccine and improve reliability of the reassortment process by eliminating gene dominance that can occur using traditional methods. The antigenicity of the vaccine viruses prepared using classical methods, and the nucleotide sequence of the HA and NA genes match the HA and NA of their respective wildtype influenza virus strains from which they were obtained. Vaccine containing a recombinant reassortant has been shown to be safe and well tolerated in a single study in children and adults. Additional safety and immunogenicity of vaccine virus prepared using the recombinant methodology is currently under investigation.

\section{ACKNOWLEDGEMENTS}

The author acknowledges with appreciation the hard work of Jean Humrich for her patience, typing and editing the manuscript.

\section{REFERENCES}

1. Thomson, D. and Thomson, R. (1934). Influenza with special reference to the complications and sequelae, bacteriology of influenzae pneumonia, pathology, epidemiological data, prevention and treatment. Ann Pickett-Thomson Res. Lab. 10, 641-677.

2. Francis, T. Jr, Quilligan, J. J. Jr and Minuse, E. (1950). Identification of another epidemic respiratory disease. Science 112, 495-497.

3. Stuart-Harris, Sir Charles and Shild, G. C. (1976). Influenza: The Viruses and the Disease. Publishing Sciences Group Inc.: Littleton, Mass.

4. Francis, T. F., Jr. (1945) Influenza: Methods of study and control. Bull. NY Acad Med. 2, 337-355.

5. Glezen. W. P. (1982). Serious morbidity and mortality associated with influenza epidemics. Epidemiol. Rev. 4, 25-44.

6. Fox, J. P., Hall, C. E., Cooney, M. K. and Foy, H. M. (1982). Influenza virus infection in Seattle families, 1975-1979. I. Study design, methods and the occurrence of infections by time and age. Am. J. Epidemiol. 116, 212-227.

7. Smith, H. and Sweet, C. (1984). Pathogenesis of influenza virus infection in ferrets, a model for human influenza. In, The Molecular Virology and Epidemiology in Influenza. ed. by C. H. Stuart-Harris and C. W. Potter. Academic Press: New York.

8. Francis, J. T. and Maassab, H. F. (1965). Influenza Viruses. In, Viral and Rickettsial Infection in Man. 4th Ed., ed. by F. L. Harsfall and I. Taruru. Lippincott: New York, 689-740.

9. Meikljohn, G., Kempe, C. H., Thalman, W. G. and Lennette, E. H. (1952). Evaluation of monovalent influenza vaccines; observations during an influenza A prime epidemic. Am. J. Hyg. 55, 12-21.

10. Couch, R. B. and Kasel, J. A. (1983). Immunity in influenza. Ann. Rev. Microbiol. 37, 529-549.

11. Ada, G. L. and Jones, P. D. (1986). The immune response to influenza virus infection. In, Options for the Control of Influenza. UCLA-ICN Symposium on Molecular and Cellular Biology, ed. by A. Kendal and P. Patriarca. New Series. Alan R. Liss: New York, Vol 36.

12. Stuart-Harris, Ch. and Schild, G. (1976). Immunity in Influenza. In, Influenza the Viruses and the Disease, ed. by C. H. Stuart-Harris and G. Schild Publishing Sciences Group: Littleton, Mass.

13. Virelizier, J. L. (1975). Host defenses against influenza virus: the role of anti-hemagglutinin antibody. J. Immunol. 115, 434-439.

14. Davenport, F. M. (1982). Influenza viruses. In, Viral Infections of Humans - Epidemiology and Control, ed. by A. S. Evans. Plenum Medical Books: New York.

15. Greenburg, M., Jacobziner, H., Pakter, J. and Weisl, B. A. G. (1958). Maternal morbidity in the epidemic of Asian influenza, New York City 197. Am J Obstet Gynecol 76, 897-902. 
16. Webster, R. G., Kendal, A. P. and Gerhard, W. (1979). Analysis of antigenic drift in recently isolated influenza A (HIN1) viruses using monoclonal antibody preparations. Virology 96, 258-264.

17. Webster, R. G., Bean, W. J., Gorman, O. T., Chambers, T. M. and Kawaoka, Y. (1992). Evolution and ecology of influenza A viruses. Microbiol Rev 56, 152-179.

18. Wiley, D. C., Wilson, I. A. and Skehel, J. J. (1981). Structural identification of the antibody-binding sites of Hong Kong influenza hemagglutinin and their involvement in antigenic variation. Nature 238, 373378.

19. Stuart-Harris, C. H. and Schild, G. (1976). The epidemiology of influenza and the epidemiology of influenza. Part II. In, Influenza, the Viruses and the Disease, ed. by C. H. Stuart-Harris and G. Schild Publishing Sciences Group: Littleton, Mass.

20. Fox, J. P., Cooney, M. K., Hall, C. E. and Foy, H. M. (1982). Influenza virus infections in Seattle families, 1975-1979. II. Pattern of infection in invaded households and relation of age and prior antibody to occurrence of infected and related illness. Am. J. Epidemiol. 116, 228-242.

21. MacKenzie, J. S. and Houghton, M. (1974). Influenza infections during pregnancy: association with congenital malformations and with subsequent neoplasms in children, and potential hazards of live virus vaccine. Bacteriol. Rev. 38, 356-370.

22. Centers for Disease Control and Prevention. (1997). Prevention and control of influenza: recommendation of the Advisory Committee on Immunization Practices (ACIP). MMWR 46(RR-9), 1-25.

23. Atmar, R. L., Bloom, K., Keitel, W. and Couch, R. B. and Greenberg, S. B. (1990). Effects of live atteunated, cold recombinant (CR) influenza virus vaccines on pulmonary function in healthy and asthmatic adults. Vaccine 8, 217-224.

24. King, J. C., Gross, P. A., Denning, C. R. et al. (1987). Comparison of live and inactivated influenza vaccine in high risk children. Vaccine 5, 234-238.

25. Salk, J. E. (1961). An interpretation of the significance of influenza virus variation for the development of an effective vaccine. International Conference on Asian Influenza. Am. Rev. Resp. Dis. suppl 83, 153-156.

26. Alexandrova, G. I., Polezhaev, F. I., Budilovsky, G. N. et al. (1984). Recombinant cold-adapted attenuated influenza A vaccines for use in children. Reactogenicity and antigenic activity of cold-adapted recombinants and analysis of isolates from the vaccinees. Infect. Immunol. 44, 734-739.

27. Maassab, H. F. (1969). Biologic and immunologic characteristics of cold-adapted influenza virus. J. Immunol. 102, 728-732.

28. Slepuskin, A. N., Bobyleva, T. K., Ruussina, A. E., Virkina, B. S., Ellengom, N. S. and Zhadanov, V. M. (1967). Evaluation of the effectiveness of large-scale vaccination against influenza in the U.S.S.R. Bull. World Health Organ. 36, 385.
29. Wright, P. F. and Karzon, D. T. (1985). Live attenuated influenza vaccines. Prog Med Virol 34, 70-88.

30. Maassab, H. F. and DeBorde, D. C. (1985). Development and characterization of cold-adapted viruses for use as live virus vaccines. Vaccine 3, 355-369.

31. Maassab, H. F. and Bryant, M. L. (1998). Live influenza virus vaccine. In, Vaccines (3rd edn), W. B. Saunders Co.: New York, Toronto. 909-928.

32. Domingo, E., Menendez-Arias, L. and Holland, J. J. (1997). RNA virus fitness. Rev. Med. Virol. 7, 87-96.

33. Maassab, H. F., Heilman, C. A. and Herlocher, M. L. (1990). Cold-adapted influenza viruses for use as live vaccine for man. In, Viral Vaccines. Advances in Biotech Proc., ed. by A. Mizrahi. Wiley-Liss A, John Wiley and Sons, Inc.: New York, Toronto, Brisbane and Singapore. 14, 203-242.

34. Maassab, H. F. and DeBorde, D. C. (1985). Development and characterization of cold-adapted viruses for use as live virus vaccine. Vaccine 3, 355-369.

35. Treanor, J. J., Mattison, R., Domyati, G. et al. (1992). Protective efficacy of combined live intranasal and inactivated influenza A virus vaccines in the elderly. Ann Intern Med 117, 625-633.

36. Gorse, G. J., Bleshe, R. B. and Munn, N. J. (1991). Superiority of live attenuated compared to inactivated influenza A virus vaccines in older, chronically ill adults. Chest 100, 977-984.

37. Belshe, R. B., Anderson, E. L., Newman, F., Swierkosz, E. M., Nugent, S., and Maassab, H. F. (1992). Immunization of infants and young children with live attenuated trivalent cold recombinant influenza $A$ H1N1 and H3N2 and B vaccine. J. Infect. Dis. 165, 727-732.

38. Johnson, P. R., Feldman, S., Thompson, J. M., Mahoney, J. D. and Wright, P. F. (1986). Immunity to infuenza A virus infection in young children: a comparison of natural infection, live cold-adapted and inactivated vaccines. J. Infect. Dis. 154, 121-126.

39. Edwards, K. M., Dupont, W. D., Westrich, M. K., Plummer, W. D. Jr, Palmer, P. S. and Wright, P. F. (1994). A randomized controlled trial of cold-adapted and inactivated vaccines for the prevention of influenza A disease. J. Infect. Dis. 169, 68-76.

40. Bryant, M. L., CAIV Research Team. Clinical progress and benefits of cold adapted influenza vaccine in children, adults, and elderly. Abstract; International Conference on Options for the Control of Influenza III. May 4-9, 1996 Cairns, Australia. In, Vaccines, W. B. Saunders Co.: Philadelphia, London, Toronto, Montreal, Sydney, Tokyo.

41. Treanor, J., for P. Glasner. Update on clinical trials of safety an efficacy of trivalent cold-adapted influenza vaccine in children and adults. Abstract; IBC's International Industry Conference on Influenza and Other Respiratory Disorders: Latest Therapeutic and Vaccine Developments. September 26-27, 1996, Washington, DC. In Vaccines, W. B. Saunders Co.: Philadelphia, London, Toronto, Montreal, Sydney, Tokyo.

42. King, J., Belshe, R., Bryant, M., NIAID VEU Study Group. Safety and immunogenicity of intranasal 
live attenuated trivalent cold-adapted influenza vaccine (CAIV-T) given as drops or spray in children. Abstract; Am. Soc. Ped. Res. May 2-6, 1997 Washington, DC. In, Vaccines, W. B. Saunders Co.: Philadelphia, London, Toronto, Montreal, Sydney, Tokyo.

43. Belshe, R., Iacuzio, D., Mendelman, P. and Wolff, M. Efficacy of a trivalent live attenuated intranasal influenza vaccine in children. Abstract; Infectious Disease Society of America 35th Annual Meeting, September 13-16, 1997, San Francisco, CA. In, N. Engl. J. of Med. Vol 338, No. 20 1405-1413: Mass Med. Soc.
44. Radecke, F. and Billetter, M. A. (1997). Reverse genetics meets the nonsegmented negative-strand RNA viruses. Rev. Med. Virol. 7: 49-63.

45. Li, Si., Rodrigues, M., Rodriguez, D. et al. (1993). Priming with recombinant influenza virus followed by administration of recombinant vaccine a virus induces CD8 + $\mathrm{T}$ cell-mediated protective immunity against malaria. Proc. Natl. Acad. Sci. USA, 90, 52175220. 